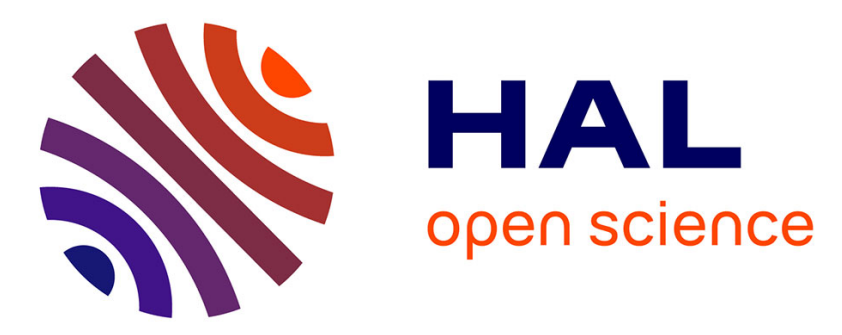

\title{
Complexity of determining the irregular chromatic index of a graph
}

Julien Bensmail

\section{To cite this version:}

Julien Bensmail. Complexity of determining the irregular chromatic index of a graph. 2013. hal$00789172 \mathrm{v} 2$

\author{
HAL Id: hal-00789172 \\ https://hal.science/hal-00789172v2
}

Submitted on 16 May 2013

HAL is a multi-disciplinary open access archive for the deposit and dissemination of scientific research documents, whether they are published or not. The documents may come from teaching and research institutions in France or abroad, or from public or private research centers.
L'archive ouverte pluridisciplinaire HAL, est destinée au dépôt et à la diffusion de documents scientifiques de niveau recherche, publiés ou non, émanant des établissements d'enseignement et de recherche français ou étrangers, des laboratoires publics ou privés. 


\title{
Complexity of determining the irregular chromatic index of a graph
}

\author{
Julien Bensmail \\ Univ. Bordeaux, LaBRI, UMR 5800, F-33400 Talence, France \\ CNRS, LaBRI, UMR 5800, F-33400 Talence, France \\ julien. bensmail@labri.fr
}

\begin{abstract}
A graph $G$ is locally irregular if adjacent vertices of $G$ have different degrees. A $k$-edge colouring $\phi$ of $G$ is locally irregular if each of the $k$ colours of $\phi$ induces a locally irregular subgraph of $G$. The irregular chromatic index $\chi_{i r r}^{\prime}(G)$ of $G$ is the least number of colours used by a locally irregular edge colouring of $G$ (if any). We show that determining whether $\chi_{i r r}^{\prime}(G)=2$ is NP-complete, even when $G$ is assumed to be a planar graph with maximum degree at most 6 .
\end{abstract}

\section{Introduction}

Let $G$ be a graph. Given a subset $F \subseteq E(G)$ of edges of $G$, the subgraph of $G$ induced by $F$ is the subgraph of $G$ whose vertices are the vertices of $G$ which are incident to edges in $F$ and whose edges are those in $F$. We say that $G$ is locally irregular if adjacent vertices of $G$ have distinct degrees.

A $k$-edge colouring $\phi: E(G) \rightarrow\{1, \ldots, k\}$ of $G$ is locally irregular if every of the $k$ colours of $\phi$ induces a locally irregular subgraph of $G$. The irregular chromatic index of $G$, denoted by $\chi_{i r r}^{\prime}(G)$, is the least number of colours needed by a locally irregular edge colouring of $G$ (if any).

The notion of locally irregular edge colouring of graphs was recently introduced by Baudon et al. [3] and combines two popular notions of graph theory, namely the ones of locally irregular graphs and adjacent vertex distinguishing edge colourings of graphs. Locally irregular graphs were first introduced under the name of highly irregular graphs in a work aiming at defining some possible ways for catching the irregularity of graphs [2].

Adjacent vertex distinguishing edge colourings of graphs have been intensively studied for the last decades (see [8] and [9] for additional informations on this topic). Numerous different such problems may thus be found in the 
literature differing mainly on what are the adopted conditions for a vertex to be considered as distinguished from its neighbours (but these conditions are related to some edge colouring). Let us introduce two examples of such problems based on a $k$-edge colouring $\phi$ of $G$ taking values in $\{1, \ldots, k\}$. In what follows, $s_{\phi}(v)$ and $m_{\phi}(v)$ denote, for some vertex $v$ of $G$, the sum and the multiset of the colours incident to $v$ by $\phi$, respectively.

If for every pair of adjacent vertex $\{u, v\}$ of $G$ we have $s_{\phi}(u) \neq s_{\phi}(v)$, then we say that $\phi$ is neighbour sum distinguishing. Observe that not all graphs admit neighbour sum distinguishing edge colourings since isolated edges do not admit any such colouring. However, graphs without such edges apart, it is believed that 3 colours are sufficient to make every pair of adjacent vertices distinguished in any graph. Karoński, Łuczak and Thomason thus raised the following elegant conjecture known as the 1-2-3 Conjecture [7].

1-2-3 Conjecture. All graphs without isolated edges admit a neighbour sum distinguishing 3-edge colouring.

If we rather have $m_{\phi}(u) \neq m_{\phi}(v)$ for every pair of adjacent vertices $\{u, v\}$ in $G$, then $\phi$ is said neighbour multiset distinguishing. Neighbour multiset distinguishing edge colourings (also called detectable colourings in the literature) were mainly considered by Addario-Berry et al. in [1]. They showed that, graphs with isolated edges apart, all graphs admit a neighbour multiset distinguishing 4-edge colouring and that a large family of graphs even admit a neighbour multiset distinguishing 3-edge colouring. These results would seem to indicate that graphs without isolated edges admit a multiset distinguishing edge colouring using at most 3 colours.

Detection Conjecture. All graphs without isolated edges admit a neighbour multiset distinguishing 3-edge colouring.

Clearly, every neighbour sum distinguishing or locally irregular edge colouring is also neighbour multiset distinguishing. Locally irregular edge colourings may thus be considered as a new tool for tackling the Detection Conjecture. However, there does not seem to be any systematic relationship between neighbour sum distinguishing and locally irregular edge colourings.

Similarly as for neighbour sum and neighbour multiset distinguishing edge colourings, there exist graphs that do not admit any locally irregular edge colouring. Such graphs are said non-colourable (with respect to locally irregular edge colourings). As shown in [3], non-colourable graphs include odd length paths and cycles, and a family of tree-like graphs with maximum degree at most 3 obtained by connecting an arbitrary number of triangles in a specific way. It is worth mentioning that, because of their simple structure, non-colourable graphs may be recognized in polynomial time. As for 
colourable graphs, all known candidates have irregular chromatic index at most 3 , and we know graphs with irregular chromatic index exactly $k$ for every $k \in\{1,2,3\}$. For example, observe that $\chi_{i r r}^{\prime}\left(P_{3}\right)=1, \chi_{i r r}^{\prime}\left(P_{2 q+1}\right)=2$ for every $q \geq 2$, and $\chi_{i r r}^{\prime}\left(C_{2 q^{\prime}}\right)=3$ for every odd $q^{\prime} \geq 3$, where $P_{n}$ and $C_{n}$ are the path and cycle on $n$ vertices, respectively, for every $n \geq 1$.

We still do not know whether there exist graphs with irregular chromatic index at least 4 . Therefore, the following conjecture was raised in [3].

Local Irregularity Conjecture. Colourable graphs have irregular chromatic index at most 3.

If the Local Irregularity Conjecture turned out to be true, then all colourable graphs would have irregular chromatic index 1,2 or 3 . So the next interesting question would be to find out whether it is easy to determine the tight irregular chromatic index of a given colourable graph. For this purpose, we introduce the following decision problem.

Locally Irregular $k$-Edge Colouring $-k$-LIEC

Instance: A graph $G$.

Question: Do we have $\chi_{i r r}^{\prime}(G) \leq k$ ?

Clearly, $\chi_{i r r}^{\prime}(G)=1$ if and only if $G$ is locally irregular itself. Since checking whether a graph is locally irregular can be done in polynomial time, 1-LIEC is in P. In this paper, we show that determining the tight irregular chromatic index of a given graph is difficult in general.

Theorem 1. 2-LIEC is NP-complete, even when restricted to planar graphs with maximum degree at most 6.

Studies of similar decision problems in the context of neighbour sum and neighbour multiset distinguishing edge colourings may be found in the literature. In particular, it was shown that deciding whether a graph admits a neighbour sum or a neighbour multiset distinguishing 2-edge colouring is NP-complete in general (see [4] and [6], respectively). Theorem 1 thus meets these two complexity results.

Section 3 is devoted to the proof of Theorem 1. We first introduce, in upcoming Section 2, some notation and graph constructions used in the proof of Theorem 1. Concluding remarks are given in Section 4 .

\section{Definitions and notation}

Let $G$ be an arbitrary graph and $\phi: E(G) \rightarrow\{0,1\}$ be a locally irregular 2-edge colouring of $G$. The subgraph of $G$ induced by colour 1 (resp. 0 ) of $\phi$ is called the 1-subgraph (resp. 0-subgraph) of $G$ induced by $\phi$. When no 

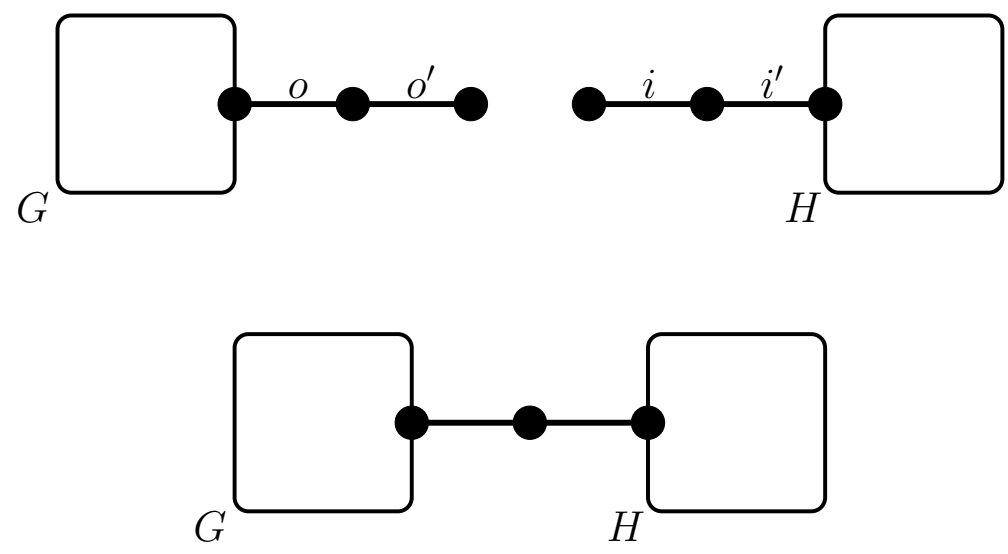

Figure 1: Two graphs $G$ and $H$ with output $\left(o, o^{\prime}\right)$ and input $\left(i, i^{\prime}\right)$, respectively, and the connection of $G$ and $H$ along $\left(o, o^{\prime}\right)$ and $\left(i, i^{\prime}\right)$

ambiguity is possible, the mention to $G$ and $\phi$ may be omitted. Given a vertex $v$ of $G$, we denote by $d_{\phi, 1}(v)$ (resp. $\left.d_{\phi, 0}(v)\right)$ the degree of the vertex $v$ in the 1- (resp. 0-) subgraph of $G$.

An input (resp. output) of $G$ is a pair of edges $\left(i, i^{\prime}\right)$ (resp. $\left.\left(o, o^{\prime}\right)\right)$ such that $i=u v$ and $i^{\prime}=v w$ (resp. $o=w v$ and $o^{\prime}=v u$ ) and $u$ and $v$ have degree 1 and 2 in $G$, respectively. Consider now two graphs $G$ and $H$ such that $\left(o, o^{\prime}\right)$ and $\left(i, i^{\prime}\right)$ are an output of $G$ and an input of $H$, respectively. The connection of $G$ and $H$ along $\left(o, o^{\prime}\right)$ and $\left(i, i^{\prime}\right)$ is the graph obtained by taking the disjoint union of $G$ and $H$, and then identifying the edges $o$ and $i$, and $o^{\prime}$ and $i^{\prime}$. The inputs and outputs of the resulting graph are those of $G$ and $H$ that have not been used for the connection. This construction is depicted in Figure 1.

Given two outputs $\left(o_{1}, o_{1}^{\prime}\right)$ and $\left(o_{2}, o_{2}^{\prime}\right)$ of $G$ with $o_{1}^{\prime}=v_{1} w_{1}$ and $o_{2}^{\prime}=$ $v_{2} w_{2}$, the identification of the two outputs $\left(o_{1}, o_{1}^{\prime}\right)$ and $\left(o_{2}, o_{2}^{\prime}\right)$ is obtained by identifying the vertices $w_{1}$ and $w_{2}$ in $G$. The identification of more than two outputs of $G$ is defined analogously. According to the original definition, notice that identified outputs of $G$ are not outputs of the resulting graph.

\section{Proof of Theorem 1}

The reader is referred to [5] for details on computational complexity theory. Given a graph $G$ and a 2-edge colouring $\phi$ of $G$, one can check in polynomial time whether the two subgraphs of $G$ induced by $\phi$ are locally irregular. Therefore, 2-LIEC is in NP.

We now show the completeness of 2-LIEC in NP by reduction from the following NP-complete problem. 


\section{1-IN-3 SAT}

Instance: A $3 \mathrm{CNF}$ formula $F$ with clauses $C_{1}, \ldots, C_{m}$ over variables $x_{1}, \ldots, x_{n}$. Question: Is there a 1-in-3 truth assignment of the variables of $F$, that is a truth assignment such that each clause of $F$ has exactly one true literal?

Let us make some observations on the structure of $F$. First, we can assume that every possible literal appears in $F$. Indeed, if $x_{i}$ does not appear in any clause of $F$, then clearly the 3CNF formula

$$
F^{\prime}=F \wedge\left(x_{i} \vee \overline{x_{i}} \vee \overline{x_{n+1}}\right) \wedge\left(x_{n+1} \vee \overline{x_{n+1}} \vee \overline{x_{n+1}}\right),
$$

where $x_{n+1}$ is a new variable, admits a 1 -in- 3 truth assignment of its variables if and only if $F$ admits one too. Since there are $2 n$ literals related to the variables of $F$, a formula equivalent to $F$ that contains every possible literal over its variables can be obtained from $F$ in polynomial time.

Next, it should be clear that a clause $\left(x_{i} \vee x_{i} \vee x_{i}\right)$ cannot be satisfied by exactly one of its literals by any truth assignment of the variables of $F$. Therefore, we may suppose that $F$ does not contain such a clause. Finally, observe that if $F$ contains a clause $\left(x_{i} \vee x_{i} \vee x_{j}\right)$, then, in every 1-in-3 truth assignment of $F$, the variables $x_{i}$ and $x_{j}$ have to be set to false and true, respectively. When dealing with such a clause, we say that $x_{i}$ and $x_{j}$ are forced to false and true, respectively.

We now explain how to get a graph $G_{F}$ from $F$ such that $F$ is satisfiable in a 1-in-3 way if and only if $\chi_{i r r}^{\prime}\left(G_{F}\right)=2$. Here is a brief sketch of the construction. The graph $G_{F}$ has to be seen as an electrical circuit through which two opposite signals, the true and the false ones, are propagated through many components whose inputs and outputs are connected in a specific way. Besides, the propagation of these signals has the same properties as the propagation of a locally irregular 2-edge colouring in a graph. The components of $G_{F}$ are the following. First, the generator gadget $G_{F}(S)$ of $G_{F}$ spreads the true signal through $m$ clause gadgets $G_{F}\left(C_{1}\right), \ldots, G_{F}\left(C_{m}\right)$, where each clause $C_{i}$ in $F$ is associated with the clause gadget $G_{F}\left(C_{i}\right)$ in $G_{F}$. The clause gadgets then modulate their input true signal, that is they can switch their input signal or not, and propagate it to the literal gadgets $G_{F}\left(\ell_{1}\right), \ldots, G_{F}\left(\ell_{2 n}\right)$ of $G_{F}$. Similarly as for the clauses of $F$ and the clause gadgets of $G_{F}$, there is a direct analogy between the literal $\ell_{i}$ and the literal gadget $G_{F}\left(\ell_{i}\right)$. The clause and literal gadgets are linked in the following way:

- if $C_{i}=\left(\ell_{i_{1}} \vee \ell_{i_{2}} \vee \ell_{i_{3}}\right)$ is a clause of $F$, then $G_{F}\left(C_{i}\right)$ is connected to $G_{F}\left(\ell_{i_{1}}\right)$ along an output of $G_{F}\left(C_{i}\right)$. If $\ell_{i_{2}} \neq \ell_{i_{1}}$, then $G_{F}\left(C_{i}\right)$ is connected to $G_{F}\left(\ell_{i_{2}}\right)$ along a second output of $G_{F}\left(C_{i}\right)$. Finally, if $\ell_{i_{3}} \neq \ell_{i_{1}}$ and $\ell_{i_{3}} \neq \ell_{i_{2}}$, then $G_{F}\left(C_{i}\right)$ is also connected to $G_{F}\left(\ell_{i_{3}}\right)$ along a third output. 
- Exactly one arbitrary output of each clause gadget spreads the true signal, while its other outputs (there are at most two of them) propagate the false one.

Each literal gadget $G_{F}\left(\ell_{i}\right)$ of $G_{F}$ has exactly one output and, according to the connection with the clause gadgets, exactly $n_{i}$ inputs, where $n_{i}$ is the number of distinct clauses in $F$ that contain the literal $\ell_{i}$ for every $i \in\{1, \ldots, 2 n\}$. The main property of a literal gadget $G_{F}\left(\ell_{i}\right)$ is that it outputs a signal if and only if the same signal comes in from its $n_{i}$ inputs. Moreover, if a given signal comes in from the $n_{i}$ inputs of $G_{F}\left(\ell_{i}\right)$, then $G_{F}\left(\ell_{i}\right)$ outputs the same signal. Finally, the outputs of the two literal gadgets $G_{F}\left(x_{i}\right)$ and $G_{F}\left(\overline{x_{i}}\right)$ are linked in such a way that the propagation of the signal is correct if and only if the two output signals are different.

Hence, we have an analogy between satisfying $F$ in a 1-in-3 way and spreading the true signal through $G_{F}$ :

- each clause $C_{i}$ in $F$ must have exactly one true literal and exactly one output of $G_{F}\left(C_{i}\right)$ must spread the true signal out,

- every literal $\ell_{i}$ must have the same truth value in all clauses it appears in and all the inputs of $G_{F}\left(\ell_{i}\right)$ must spread the same signal in,

- a variable $x_{i}$ and its negation $\overline{x_{i}}$ must have distinct truth values and the outputs of $G_{F}\left(x_{i}\right)$ and $G_{F}\left(\overline{x_{i}}\right)$ must spread different signals out.

We now go into the details of the proof by introducing the generator, clause and literal gadgets of $G_{F}$. The graph $G_{F}$ is constructed step by step as it is augmented by connecting these gadgets. All along this proof, the function $\phi: E\left(G_{F}\right) \rightarrow\{0,1\}$ is a locally irregular 2-edge colouring of $G_{F}$ propagated along the edges of $G_{F}$. When augmenting $G_{F}$ with a new gadget, it should be understood that $\phi$ is extended to this new gadget according to the lemmas we point out. The true (resp. false) signal is depicted as thick (resp. thin) edges in our schemas and corresponds to colour 1 (resp. 0) of $\phi$.

The generator gadget $G_{F}(S)$ of $G_{F}$ is obtained by connecting several copies of the graph $G^{*}$ depicted in Figure 2, where $\left(u_{1} u_{2}, u_{2} u_{3}\right)$ is the input of $G^{*}$, and $\left(u_{10} u_{11}, u_{11} u_{12}\right)$ and $\left(u_{19} u_{20}, u_{20} u_{21}\right)$ are the two outputs of $G^{*}$. This gadget $G^{*}$ has the following property.

Lemma 1. In a locally irregular 2-edge colouring of $G^{*}$, the input and output edges of $G^{*}$ have the same colour. 


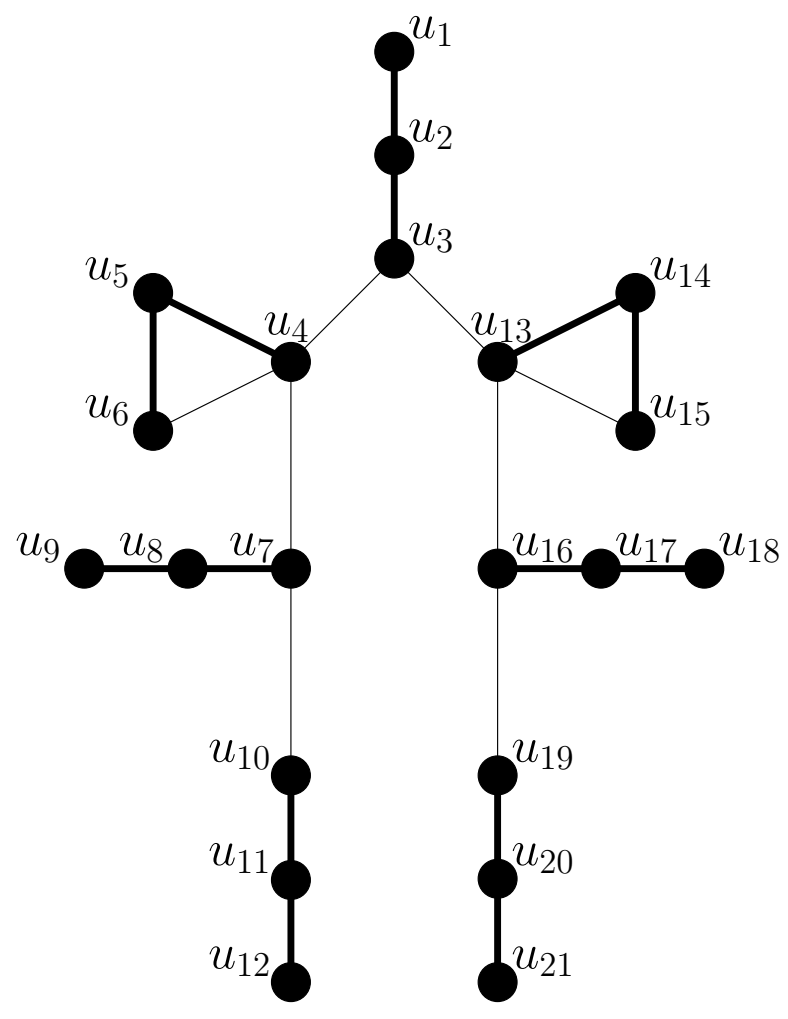

Figure 2: The gadget $G^{*}$ and a locally irregular 2-edge colouring of $G^{*}$

Proof. We initiate the locally irregular 2-edge colouring $\phi$ of $G^{*}$ with the input of $G^{*}$. Let us suppose, without loss of generality, that $\phi\left(u_{1} u_{2}\right)=$ 1. Then we have $\phi\left(u_{2} u_{3}\right)=1$ since otherwise we would have $d_{\phi, 1}\left(u_{1}\right)=$ $d_{\phi, 1}\left(u_{2}\right)=1$. We then have $\phi\left(u_{3} u_{4}\right)=\phi\left(u_{3} u_{13}\right)$ since, otherwise, we would have $d_{\phi, 1}\left(u_{2}\right)=d_{\phi, 1}\left(u_{3}\right)=2$.

Let us first suppose $\phi\left(u_{3} u_{4}\right)=\phi\left(u_{3} u_{13}\right)=1$. Clearly, we cannot have $\phi\left(u_{4} u_{5}\right)=\phi\left(u_{4} u_{6}\right)$ since, in this case, when colouring $u_{5} u_{6}$ we would have either $d_{\phi, 1}\left(u_{5}\right)=d_{\phi, 1}\left(u_{6}\right)$ or $d_{\phi, 0}\left(u_{5}\right)=d_{\phi, 0}\left(u_{6}\right)$. Suppose then $\phi\left(u_{4} u_{5}\right)=1$ and $\phi\left(u_{4} u_{6}\right)=0$. Now observe that if $\phi\left(u_{5} u_{6}\right)=1$, then we must set $\phi\left(u_{4} u_{7}\right)=0$ to get $d_{\phi, 0}\left(u_{6}\right) \neq d_{\phi, 0}\left(u_{4}\right)$. But then $d_{\phi, 1}\left(u_{4}\right)=d_{\phi, 1}\left(u_{5}\right)=2$. Similarly, if $\phi\left(u_{5} u_{6}\right)=0$, then we must have $\phi\left(u_{4} u_{7}\right)=1$ since otherwise we would have $d_{\phi, 0}\left(u_{6}\right)=d_{\phi, 0}\left(u_{4}\right)=2$. But we now have $d_{\phi, 1}\left(u_{3}\right)=d_{\phi, 1}\left(u_{4}\right)=$ 3 . Hence, we cannot extend $\phi$ to $G^{*}$ if $\phi\left(u_{3} u_{4}\right)=\phi\left(u_{3} u_{13}\right)=1$.

Thus, we must have $\phi\left(u_{3} u_{4}\right)=\phi\left(u_{3} u_{13}\right)=0$. For the same reasons as before, we have $\phi\left(u_{4} u_{5}\right)=1$ and $\phi\left(u_{4} u_{6}\right)=0$ without loss of generality. Now, if $\phi\left(u_{5} u_{6}\right)=0$, then we must have $\phi\left(u_{4} u_{7}\right)=0$ too so that $d_{\phi, 0}\left(u_{4}\right) \neq$ $d_{\phi, 0}\left(u_{6}\right)$. But then we get $d_{\phi, 1}\left(u_{4}\right)=d_{\phi, 1}\left(u_{5}\right)=1$. Therefore, we must set $\phi\left(u_{5} u_{6}\right)=1$. To ensure that $u_{4}$ and $u_{3}$ have distinct degrees in the 0 -subgraph, we now need $\phi\left(u_{4} u_{7}\right)=0$. Similarly as before, we must have 
$\phi\left(u_{9} u_{8}\right)=\phi\left(u_{8} u_{7}\right)$. Clearly, if $\phi\left(u_{9} u_{8}\right)=\phi\left(u_{8} u_{7}\right)=0$, then we must have $\phi\left(u_{7} u_{10}\right)=0$ so that $d_{\phi, 0}\left(u_{7}\right) \neq d_{\phi, 0}\left(u_{8}\right)$; but then we get $d_{\phi, 0}\left(u_{4}\right)=$ $d_{\phi, 0}\left(u_{7}\right)=3$. So $\phi\left(u_{9} u_{8}\right)=\phi\left(u_{8} u_{7}\right)=1$, and $\phi\left(u_{7} u_{10}\right)=0$ since otherwise we would have $d_{\phi, 1}\left(u_{8}\right)=d_{\phi, 1}\left(u_{7}\right)=2$. Because $d_{\phi, 0}\left(u_{7}\right)=2$, we must have $\phi\left(u_{10} u_{11}\right)=1$. Besides, to get $d_{\phi, 1}\left(u_{10}\right) \neq d_{\phi, 1}\left(u_{11}\right)$, we have to set $\phi\left(u_{11} u_{12}\right)=1$.

The locally irregular 2-edge colouring $\phi$ is propagated to the remaining edges of $G^{*}$ in a symmetric way. We finally get that the input and outputs of $G^{*}$ have the same colour via $\phi$, as claimed.

According to Lemma 1, the gadget $G^{*}$ propagates its input colour, say the thick one, of a locally irregular 2-edge colouring towards two directions. Moreover, observe that by connecting two copies $G_{1}$ and $G_{2}$ of $G^{*}$ along one output of $G_{1}$ and the input of $G_{2}$, we obtain a propagation of the thick colour into three directions. By successively repeating this construction, one can spread the thick colour towards an arbitrary number of directions. Now consider a path $P_{5}=u_{1} u_{2} u_{3} u_{4} u_{5}$ on 5 vertices with input $\left(u_{1} u_{2}, u_{2} u_{3}\right)$ and output $\left(u_{3} u_{4}, u_{4} u_{5}\right)$, and denote by $G^{\prime}$ the graph obtained by connecting $G^{*}$ and $P_{5}$ along $\left(u_{10} u_{11}, u_{11} u_{12}\right)$ and $\left(u_{1} u_{2}, u_{2} u_{3}\right)$. Clearly, in every extension of a locally irregular 2-edge colouring of $G^{*}$ to $G^{\prime}$, the two new output edges $u_{3} u_{4}$ and $u_{4} u_{5}$ of $G^{\prime}$ are coloured with the thin colour. Hence, we may also spread the thin colour as well.

The generator gadget $G_{F}(S)$ of $G_{F}$ is obtained by combining the previous remarks, that is by connecting several copies of $G^{*}$ and $P_{5}$. From now, we suppose that $\phi$ is the locally irregular 2-edge colouring of $G_{F}$ mentioned before. This colouring is initiated with $G_{F}(S)$ in such a way that its input is coloured 1. According to our terminology, $G_{F}(S)$ has a finite number of outputs which are either positive or negative, depending on whether they are coloured 1 or 0 via $\phi$, respectively. This number of outputs will be clarified at the end of this proof.

In what follows, a $\left(k_{1}, k_{2}\right)$-vertex $a$ of $G_{F}$ for some $k_{1}, k_{2} \geq 1$ is the vertex of degree $k_{1}+k_{2}$ resulting from the identification of $k_{1}$ distinct positive outputs of $G_{F}(S)$ and $k_{2}$ distinct negative outputs of $G_{F}(S)$. Remark that this resulting vertex $a$ has degree $k_{1}$ (resp. $k_{2}$ ) in the 1- (resp. 0-) subgraph of $G_{F}(S)$. According to our terminology, for such a vertex we have $d_{\phi, 1}(a)=k_{1}$ and $d_{\phi, 0}(a)=k_{2}$. Besides, it should be understood that a subgraph of $G_{F}$ containing a $\left(k_{1}, k_{2}\right)$-vertex is implicitly connected to $G_{F}(S)$.

We now introduce the clause gadgets $G_{F}\left(C_{1}\right), \ldots, G_{F}\left(C_{m}\right)$ that are connected to some outputs of $G_{F}(S)$. Depending on the number $c_{i} \in\{2,3\}$ of distinct literals in $C_{i}$ (recall that $c_{i} \neq 1$ ), the clause gadget $G_{F}\left(C_{i}\right)$ can be of two different forms.

- If $c_{i}=2$, then $G_{F}\left(C_{i}\right)$ is the graph depicted in Figure 3.a, obtained 


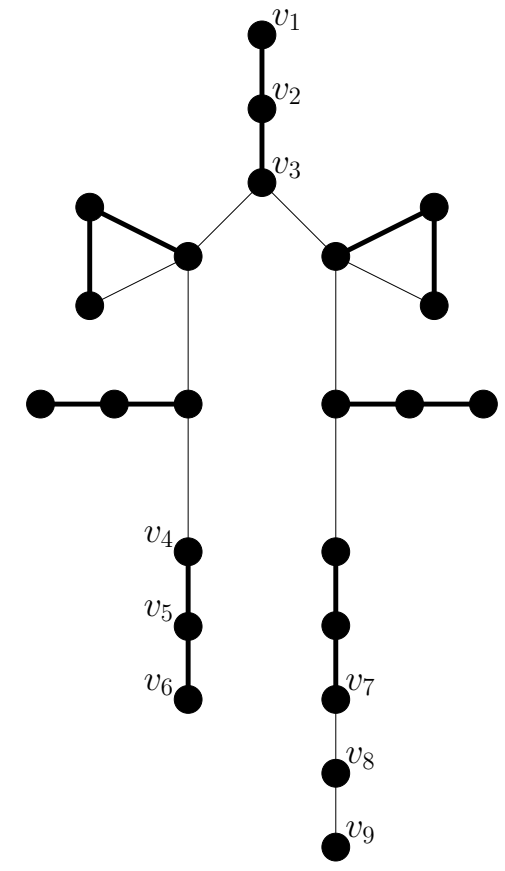

(a) Case where $c_{i}=2$

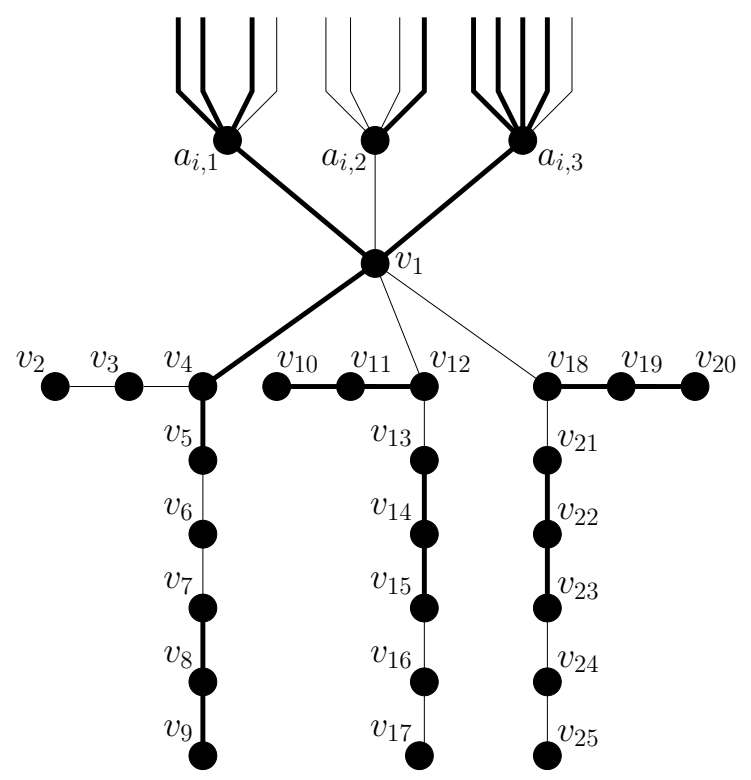

(b) Case where $c_{i}=3$

Figure 3: The clause gadget $G_{F}\left(C_{i}\right)$ and locally irregular 2-edge colourings of $G_{F}\left(C_{i}\right)$

by connecting a copy of $G^{*}$ and $P_{5}$ in such a way that $G_{F}\left(C_{i}\right)$ has one positive output and one negative output. It is connected to $G_{F}(S)$ along one positive output of $G_{F}(S)$ and $\left(v_{1} v_{2}, v_{2} v_{3}\right)$, where $\left(v_{1} v_{2}, v_{2} v_{3}\right)$ is the input of $G_{F}\left(C_{i}\right)$.

- If $c_{i}=3$, then $G_{F}\left(C_{i}\right)$ is a copy of the graph depicted in Figure 3.b, where $a_{i, 1}, a_{i, 2}$ and $a_{i, 3}$ are $(3,1)$-, $(1,3)$ - and $(4,1)$-vertices, respectively, and $\left(v_{7} v_{8}, v_{8} v_{9}\right),\left(v_{15} v_{16}, v_{16} v_{17}\right)$ and $\left(v_{23} v_{24}, v_{24} v_{25}\right)$ are its three outputs.

When $c_{i}=3$, the clause gadget $G_{F}\left(C_{i}\right)$ has the following property.

Lemma 2. Suppose $c_{i}=3$. In any extension of $\phi$ to $G_{F}\left(C_{i}\right)$, exactly one output of $G_{F}\left(C_{i}\right)$ is coloured 1 while all of its other outputs are coloured 0.

Proof. Since $a_{i, 1}$ is adjacent to a vertex of $G_{F}(S)$ with degree 2 in the 0subgraph, we must set $\phi\left(a_{i, 1} v_{1}\right)=1$. For the same reason, we have to set $\phi\left(a_{i, 2} v_{1}\right)=0$ and $\phi\left(a_{i, 3} v_{1}\right)=1$. So we get $d_{\phi, 1}\left(a_{i, 1}\right)=d_{\phi, 0}\left(a_{i, 2}\right)=$ 4 and $d_{\phi, 1}\left(a_{i, 3}\right)=5$. Observe that if no edge, two edges or three edges among those in $\left\{v_{1} v_{4}, v_{1} v_{12}, v_{1} v_{18}\right\}$ were coloured 1 via $\phi$ and the other ones were coloured 0 , then we would get $d_{\phi, 0}\left(v_{1}\right)=d_{\phi, 0}\left(a_{i, 2}\right), d_{\phi, 1}\left(v_{1}\right)=$ 
$d_{\phi, 1}\left(a_{i, 1}\right)$ or $d_{\phi, 1}\left(v_{1}\right)=d_{\phi, 1}\left(a_{i, 3}\right)$, respectively. Therefore, exactly one edge in $\left\{v_{1} v_{4}, v_{1} v_{12}, v_{1} v_{18}\right\}$ is coloured 1 via $\phi$, while the other two are coloured 0 .

Let us suppose $\phi\left(v_{1} v_{4}\right)=1$ without loss of generality. Observe that we have $d_{\phi, 1}\left(v_{1}\right)=d_{\phi, 0}\left(v_{1}\right)=3$. Once again, the edges $v_{2} v_{3}$ and $v_{3} v_{4}$ have to be coloured with the same colour, but this cannot be 1 . Indeed, if $\phi\left(v_{2} v_{3}\right)=\phi\left(v_{3} v_{4}\right)=1$, then we have $d_{\phi, 1}\left(v_{3}\right)=d_{\phi, 1}\left(v_{4}\right)=2$. Thus, we have to set $\phi\left(v_{4} v_{5}\right)=1$ but then we get $d_{\phi, 1}\left(v_{4}\right)=d_{\phi, 1}\left(v_{1}\right)=3$. So we necessarily have $\phi\left(v_{2} v_{3}\right)=\phi\left(v_{3} v_{4}\right)=0$, and $\phi\left(v_{4} v_{5}\right)=1$ since otherwise we would get $d_{\phi, 0}\left(v_{3}\right)=d_{\phi, 0}\left(v_{4}\right)=2$. Now, because $d_{\phi, 1}\left(v_{4}\right)=2$, we need $\phi\left(v_{5} v_{6}\right)=\phi\left(v_{6} v_{7}\right)=0$. Analogously, we have $\phi\left(v_{7} v_{8}\right)=\phi\left(v_{8} v_{9}\right)=1$.

Repeating the same arguments towards $v_{17}$ and $v_{25}$, knowing that $\phi\left(v_{1} v_{12}\right)=$ $\phi\left(v_{1} v_{18}\right)=0$, we get $\phi\left(v_{15} v_{16}\right)=\phi\left(v_{16} v_{17}\right)=0$ and $\phi\left(v_{23} v_{24}\right)=\phi\left(v_{24} v_{25}\right)=$ 0 . Finally observe that this colouring of $G_{F}\left(C_{i}\right)$ via $\phi$ is not unique and depends on which edge from $\left\{v_{1} v_{4}, v_{1} v_{12}, v_{1} v_{18}\right\}$ is coloured 1 . But in every extension of $\phi$ to $G_{F}\left(C_{i}\right)$, we get that exactly one input of $G_{F}\left(C_{i}\right)$ is coloured 1.

We finally clarify the literal gadgets. Recall that for $i \in\{1, \ldots, 2 n\}$, we denote by $n_{i}$ the number of distinct clauses of $F$ that contains $\ell_{i}$. Besides, we have $n_{i} \geq 1$ for every such integer by assumption. The outputs of the clause gadgets $G_{F}\left(C_{1}\right), \ldots, G_{F}\left(C_{m}\right)$ are now connected with the literal gadgets $G_{F}\left(\ell_{1}\right), \ldots, G_{F}\left(\ell_{2 n}\right)$ of $G_{F}$ as follows. The literal gadget $G_{F}\left(\ell_{i}\right)$ has one output and exactly $n_{i}$ inputs connected to $n_{i}$ clause gadgets of $G_{F}$ according to which clauses of $F$ contain $\ell_{i}$. More precisely, if $\ell_{i}$ is contained at least once in $C_{j}$, then we connect $G_{F}\left(C_{j}\right)$ and $G_{F}\left(\ell_{i}\right)$ along exactly one output of $G_{F}\left(C_{j}\right)$ and one input of $G_{F}\left(\ell_{i}\right)$. The output of $G_{F}\left(C_{j}\right)$ used for the connection is chosen arbitrarily, except in the case where $c_{j}=2$. Recall that, in this case, $C_{j}$ has two distinct literals $x_{i}$ and $x_{i}^{\prime}$ that are forced to false and true, respectively. To model this constraint, we force one input of $G_{F}\left(x_{i}\right)$ to be coloured 0 while one input of $G_{F}\left(x_{i}^{\prime}\right)$ is coloured 1. This is done by using a negative and a positive output of $G_{F}\left(C_{j}\right)$, respectively, for the connection of $G_{F}\left(C_{j}\right)$ and $G_{F}\left(x_{i}\right)$, and $G_{F}\left(C_{j}\right)$ and $G_{F}\left(x_{i}^{\prime}\right)$.

The structure of the literal gadget $G_{F}\left(\ell_{i}\right)$ depends on the value of $n_{i}$ :

- if $n_{i}=1$, then $G_{F}\left(\ell_{i}\right)$ is a copy of $P_{7}=u_{1} u_{2} \ldots u_{6} u_{7}$ and has input $\left(u_{1} u_{2}, u_{2} u_{3}\right)$ and output $\left(u_{5} u_{6}, u_{6} u_{7}\right)$,

- if $n_{i}=2$, then $G_{F}\left(\ell_{i}\right)$ is a copy of the graph depicted in Figure 4.a, whose inputs are $\left(w_{1} w_{2}, w_{2} w_{5}\right)$ and $\left(w_{3} w_{4}, w_{4} w_{5}\right)$, and whose output is $\left(w_{11} w_{12}, w_{12} w_{13}\right)$,

- if $n_{i} \geq 3$, then $G_{F}\left(\ell_{i}\right)$ is a copy of the graph depicted in Figure 4.b, whose inputs are $\left(i_{1} i_{1}^{\prime}, i_{1}^{\prime} w_{1}\right), \ldots,\left(i_{n_{i}} i_{n_{i}}^{\prime}, i_{n_{i}}^{\prime} w_{1}\right)$ and whose output is 


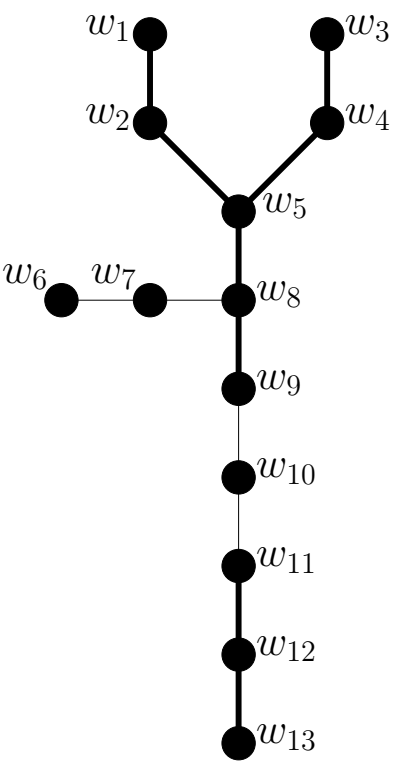

(a) Case where $n_{i}=2$

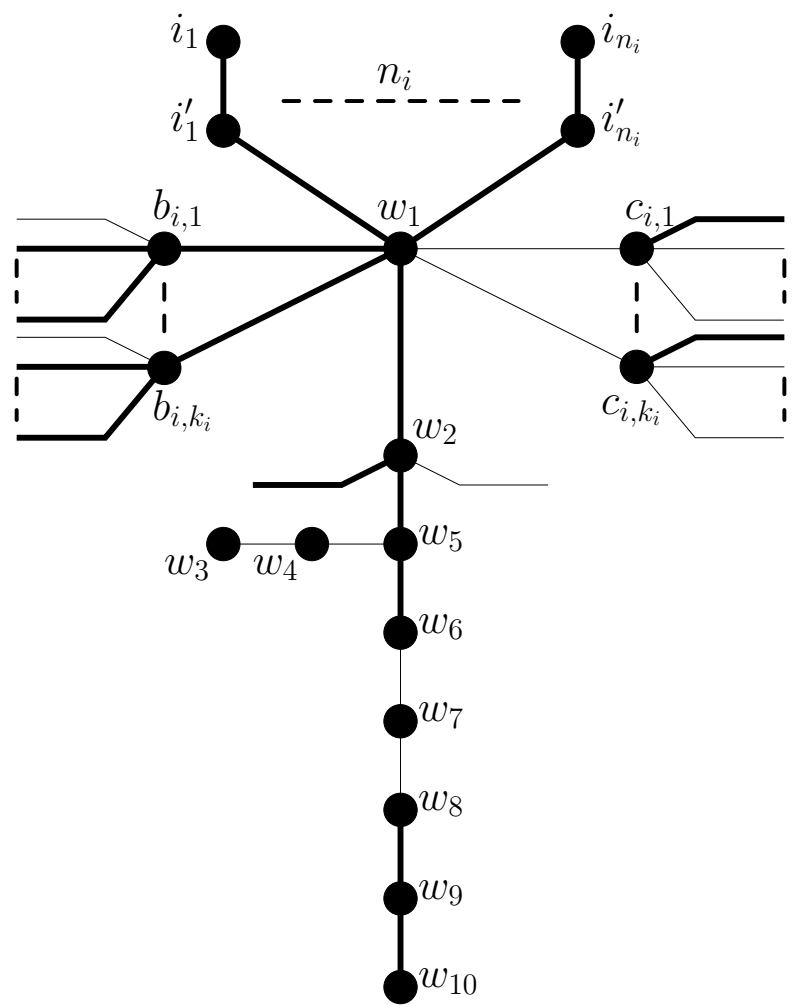

(b) Case where $n_{i} \geq 3$

Figure 4: The literal gadget $G_{F}\left(\ell_{i}\right)$ in the cases where $n_{i} \geq 2$ and locally irregular 2-edge colourings of $G_{F}\left(\ell_{i}\right)$

$\left(w_{8} w_{9}, w_{9} w_{10}\right)$. In this gadget, the vertices $b_{i, 1}, \ldots, b_{i, k_{i}}\left(\operatorname{resp} . c_{i, 1}, \ldots, c_{i, k_{i}}\right)$, where $k_{i}=\left\lfloor\frac{n_{i}}{2}\right\rfloor+1$, are $\left(n_{i}, 1\right)-,\left(n_{i}+1,1\right)-, \ldots,\left(n_{i}+\left\lfloor\frac{n_{i}}{2}\right\rfloor, 1\right)$-vertices (resp. $\left(1, n_{i}\right)-,\left(1, n_{i}+1\right)-, \ldots,\left(1, n_{i}+\left\lfloor\frac{n_{i}}{2}\right\rfloor\right)$-vertices), respectively. Besides, in this gadget, $w_{2}$ results from the identification of a positive output and a negative output of $G_{F}(S)$.

When $n_{i}=2$ or $n_{i} \geq 3$, the important property of $G_{F}\left(\ell_{i}\right)$ is that $\phi$ can be propagated to $G_{F}\left(\ell_{i}\right)$ if and only if all of its inputs have the same colour. This is proved in the following two lemmas.

Lemma 3. Suppose $n_{i}=2$. The colouring $\phi$ is extendible to $G_{F}\left(\ell_{i}\right)$ if and only if all the inputs of $G_{F}\left(\ell_{i}\right)$ have the same colour. Moreover, in any extension of $\phi$ to $G_{F}\left(\ell_{i}\right)$, the output of $G_{F}\left(\ell_{i}\right)$ is coloured with the input colour of $G_{F}\left(\ell_{i}\right)$.

Proof. Suppose $\phi\left(w_{1} w_{2}\right)=\phi\left(w_{2} w_{5}\right)=1$ and $\phi\left(w_{3} w_{4}\right)=\phi\left(w_{4} w_{5}\right)=0$ without loss of generality. Observe that if $\phi\left(w_{5} w_{8}\right)=1$, then $d_{\phi, 1}\left(w_{2}\right)=$ 
$d_{\phi, 1}\left(w_{5}\right)=2$. Similarly, if $\phi\left(w_{5} w_{8}\right)=0$, then $d_{\phi, 0}\left(w_{4}\right)=d_{\phi, 0}\left(w_{5}\right)=2$. Thus $\phi$ is only extendible to $G_{F}\left(\ell_{i}\right)$ when $\phi\left(w_{1} w_{2}\right)=\phi\left(w_{2} w_{5}\right)=\phi\left(w_{3} w_{4}\right)=$ $\phi\left(w_{4} w_{5}\right)$.

Let us suppose $\phi\left(w_{1} w_{2}\right)=\phi\left(w_{2} w_{5}\right)=\phi\left(w_{3} w_{4}\right)=\phi\left(w_{4} w_{5}\right)=1$ without loss of generality. Then, we have to set $\phi\left(w_{5} w_{8}\right)=1$ since otherwise we would have $d_{\phi, 1}\left(w_{2}\right)=d_{\phi, 1}\left(w_{5}\right)=d_{\phi, 1}\left(w_{4}\right)=2$. For the same reasons as before, we necessarily have $\phi\left(w_{6} w_{7}\right)=\phi\left(w_{7} w_{8}\right)$. If this colour is 1 , then we need $\phi\left(w_{8} w_{9}\right)=1$ to distinguish $w_{7}$ and $w_{8}$ in the 1-subgraph, but then $d_{\phi, 1}\left(w_{5}\right)=d_{\phi, 1}\left(w_{8}\right)=3$. So, $\phi\left(w_{6} w_{7}\right)=\phi\left(w_{7} w_{8}\right)=0$ and we need to set $\phi\left(w_{8} w_{9}\right)=1$ since otherwise the 0-subgraph would have two adjacent vertices with degree 2 . Since $d_{\phi, 1}\left(w_{8}\right)=2$, the colouring $\phi$ is propagated alternatively along the path $w_{9} w_{10} w_{11} w_{12} w_{13}$ in such a way that $\phi\left(w_{9} w_{10}\right)=\phi\left(w_{10} w_{11}\right)=0$ and $\phi\left(w_{11} w_{12}\right)=\phi\left(w_{12} w_{13}\right)=1$.

We show that the same property holds for every $n_{i} \geq 3$.

Lemma 4. Suppose $n_{i} \geq 3$. The colouring $\phi$ is extendible to $G_{F}\left(\ell_{i}\right)$ if and only if all the inputs of $G_{F}\left(\ell_{i}\right)$ have the same colour. Moreover, in any extension of $\phi$ to $G_{F}\left(\ell_{i}\right)$, the output of $G_{F}\left(\ell_{i}\right)$ is coloured with the input colour of $G_{F}\left(\ell_{i}\right)$.

Proof. For the same reasons as before, the edges $b_{i, 1} w_{1}, \ldots, b_{i, k_{i}} w_{1}$ (resp. $c_{i, 1} w_{1}, \ldots, c_{i, k_{i}} w_{1}$ ) have to be coloured 1 (resp. 0) in any extension of $\phi$ to $G_{F}\left(\ell_{i}\right)$. Therefore, the vertex $w_{1}$ is adjacent to vertices with degree $n_{i}+1, n_{i}+2, \ldots, n_{i}+\left\lfloor\frac{n_{i}}{2}\right\rfloor+1$ in both the 1 - and the 0 -subgraphs. Let us now suppose that $y$ inputs of $G_{F}\left(\ell_{i}\right)$ are coloured 1 via $\phi$, where $\left\lceil\frac{n_{i}}{2}\right\rceil \leq y \leq n_{i}-1$. Then $w_{1}$ has degree $y+k_{i}\left(\right.$ if $\left.\phi\left(w_{1} w_{4}\right)=0\right)$ or $y+k_{i}+1$ (otherwise) in the 1 -subgraph, but these two values belong to the set $\left\{n_{i}+1, \ldots, n_{i}+\left\lfloor\frac{n_{i}}{2}\right\rfloor+1\right\}$. Therefore, all the inputs of $G_{F}\left(\ell_{i}\right)$ must have the same colour via $\phi$. Let us suppose that this colour is 1 without loss of generality. Then, we also necessarily have $\phi\left(w_{1} w_{2}\right)=1$ since otherwise we would have $d_{\phi, 1}\left(w_{1}\right)=$ $d_{\phi, 1}\left(b_{i, k_{i}}\right)=n_{i}+\left\lfloor\frac{n_{i}}{2}\right\rfloor+1$. Observe that so far, the 0 -subgraph is locally irregular since $k_{i}<n_{i}+1$ for every $n_{i} \geq 3$.

Since $w_{2}$ has degree 2 in the 1 -subgraph and is already adjacent to a vertex with degree 2 in the 1 -subgraph, we need to set $\phi\left(w_{2} w_{5}\right)=1$. Now observe that if we set $\phi\left(w_{3} w_{4}\right)=\phi\left(w_{4} w_{5}\right)=1$, then we need $\phi\left(w_{5} w_{6}\right)=1$ so that $w_{4}$ and $w_{5}$ does not have the same degree in the 1-subgraph. But then we have $d_{\phi, 1}\left(w_{5}\right)=d_{\phi, 1}\left(w_{2}\right)=3$. Thus, $\phi\left(w_{3} w_{4}\right)=\phi\left(w_{4} w_{5}\right)=0$ and $\phi\left(w_{5} w_{6}\right)=1$ since otherwise $w_{4}$ and $w_{5}$ would have degree 2 in the 0 subgraph. Now, $w_{5}$ has degree 2 in the 1-subgraph and, thus, the colouring must alternate along $w_{6} w_{7} w_{8} w_{9} w_{10}$. For similar reasons as before, this is done in such a way that $\phi\left(w_{8} w_{9}\right)=\phi\left(w_{9} w_{10}\right)=1$. 


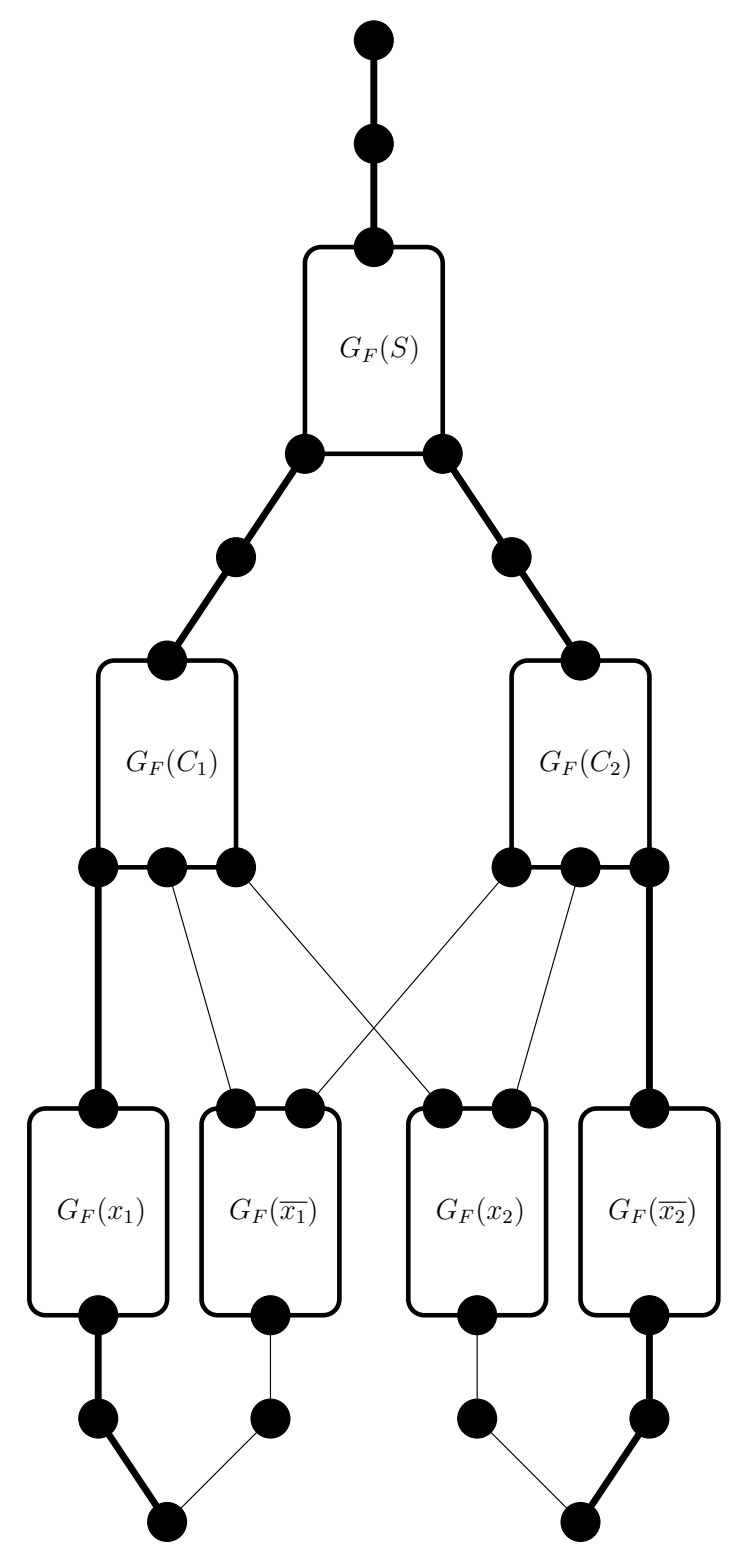

Figure 5: The reduced graph $G_{F}$ obtained from $F=\left(x_{1} \vee \overline{x_{1}} \vee x_{2}\right) \wedge\left(\overline{x_{1}} \vee\right.$ $x_{2} \vee \overline{x_{2}}$ ) and a locally irregular 2-edge colouring of $G_{F}$ implying that $F$ is satisfiable in a 1-in-3 way for $x_{1}=1$ and $x_{2}=0$. All the outputs of $G_{F}(S)$ used to force the propagation of the two colours along $G_{F}$ are not represented.

Finally, for each variable $x_{i}$ in $F$, we identify the outputs of the literal gadgets $G_{F}\left(x_{i}\right)$ and $G_{F}\left(\overline{x_{i}}\right)$. Observe that $\phi$ is a locally irregular 2-edge colouring of $G_{F}$ if and only if the output of $G_{F}\left(x_{i}\right)$ is coloured with a colour different from the one used to colour the output of $G_{F}\left(\overline{x_{i}}\right)$. Indeed, if this 
is not the case, then vertices with degree 2 would be adjacent in either the 1- of the 0-subgraph. A schematic example of a graph resulting from this reduction is represented in Figure 5.

To show that this reduction may be achieved in polynomial time, we now determine the number of vertices of $G_{F}$. The number of outputs of $G_{F}(S)$ may be computed as follows.

- In the worst case, a clause $C_{i}$ has three distinct literals and, in this special case, the clause gadget $G_{F}\left(C_{i}\right)$ contains a $(3,1)$-, a $(1,3)$ - and a $(4,1)$-vertex. Thus, we need $O(m)$ distinct outputs of $G_{F}(S)$ to construct the clause gadgets of $G_{F}$.

- We have $n_{i} \leq m$ for every $i \in\{1, \ldots, 2 n\}$. Thus, in the worst case, the literal gadget $G_{F}\left(\ell_{i}\right)$ needs $2 k_{i}$ special vertices constructed thanks to $O\left(m^{2}\right)$ dedicated outputs of $G_{F}(S)$. Since there are $2 n$ literals in $F$, we get that $O\left(n m^{2}\right)$ outputs of $G_{F}(S)$ may be necessary to construct the literal gadgets of $G_{F}$.

Thus, $O\left(\mathrm{~nm}^{2}\right)$ outputs of $G_{F}(S)$ are necessary to ensure that $\phi$ is propagated correctly along $G_{F}$. These are obtained by connecting $O\left(\mathrm{~nm}^{2}\right)$ copies of $G^{*}$, while $G^{*}$ has a constant number of vertices. Roughly, omitting that some outputs of $G_{F}(S)$ are negative, we get that the order of $G_{F}(S)$ is $O\left(n m^{2}\right)$.

Finally, the number of vertices of a clause or literal gadget of $G_{F}$ that do not belong to the generator gadget is clearly upper bounded by $O(m+$ $n)$. Therefore, the number of new vertices needed to construct the clause and literal gadgets of $G_{F}$, that is vertices that do not belong to $G_{F}(S)$, is irrelevant compared to $O\left(\mathrm{~nm}^{2}\right)$.

According to the previous arguments, it is clear that this reduction is achieved in polynomial time regarding the size of $F$.

\section{Discussion}

Note that if the Local Irregularity Conjecture were true, then any colourable graph would have irregular chromatic index at most $k$ for every $k \geq 3$, and, for such a value of $k$, the problem $k$-LIEC would thus be equivalent to the one of determining whether $G$ is colourable. Thanks to the full characterization of non-colourable graphs exhibited in [3], non-colourable graphs may be recognized in polynomial time. Hence, if the Local Irregularity Conjecture were true, then we would get that $k$-LIEC is NP-complete when $k=2$, and is in $\mathrm{P}$ otherwise. 
It is also worth mentioning that Theorem 1 implies that there should not exist an FPT algorithm for $k$-LIEC parameterised by $k$. Indeed, such an algorithm, applied to the case $k=2$, would lead to a polynomial-time algorithm for deciding whether the irregular chromatic index of a graph is 2, contradicting Theorem 1 unless $\mathrm{P}=\mathrm{NP}$.

Note that the only unavoidable edge crossings in a reduced graph $G_{F}$ only concern edges between clause and literal gadgets. We can directly get rid of these crossings by assuming that $F$ is a planar formula. Since 1-IN-3 $\mathrm{SAT}$ is known to remain NP-complete when restricted to planar formula, we get that 2-LIEC is NP-hard when restricted to planar graphs.

In $G_{F}$, the vertices with the largest degrees are those from the literal gadgets whose associated literals appear in at least 3 clauses. Note that we could replace such gadgets by several copies of the gadget depicted in Figure 4.a, so that literal gadgets all have maximum degree 3 . Proceed as follows for a gadget $G_{F}\left(\ell_{i}\right)$. Recall that $n_{i}$ outputs $\left(o_{1}, o_{1}^{\prime}\right), \ldots,\left(o_{n_{i}}, o_{n_{i}}^{\prime}\right)$ of the clause gadgets must propagate the colouring to $G_{F}\left(\ell_{i}\right)$. First take one copy $G_{1}$ of the graph of Figure 4.a, and connected $\left(o_{1}, o_{1}^{\prime}\right)$ and $\left(o_{2}, o_{2}^{\prime}\right)$ with the inputs of $G_{1}$, respectively. According to Lemma 3, the colouring is propagated to the output of $G_{1}$, say $o\left(G_{1}\right)$ and $o^{\prime}\left(G_{1}\right)$, if and only if $\left(o_{1}, o_{1}^{\prime}\right)$ and $\left(o_{2}, o_{2}^{\prime}\right)$ have the same colour. Besides, if the colouring is propagated, then $o\left(G_{1}\right)$ and $o^{\prime}\left(G_{1}\right)$ have the same colour as the input edges. Now, take another copy $G_{2}$ of the graph of Figure 4.a, and connect its inputs with the output of $G_{1}$, and $\left(o_{3}, o_{3}^{\prime}\right)$, respectively. Once again, the colouring is propagated if and only if the two input colours are the same. And so on.

Using the gadget obtained in this way by using $n_{i}-1$ copies of the gadget from Figure 4.a is thus equivalent as using the gadget from Figure 4.b. If any two of the outputs $\left(o_{1}, o_{1}^{\prime}\right), \ldots,\left(o_{n_{i}}, o_{n_{i}}^{\prime}\right)$ have distinct colours, then the colouring cannot be propagated. Therefore, the literal gadgets of $G_{F}$ can all have maximum degree 3 . Under this assumption, the maximum degree of any reduced graph $G_{F}$ is at most 6 , which is the maximum degree of a clause gadget whose associated clause of $F$ have three distinct literals. We thus get that 2-LIEC remains NP-hard when restricted to graphs with maximum degree at most 6 .

Observe that, in the proof of Theorem 1, the resulting graph $G_{F}$ is not bipartite mainly because of the induced triangles of the gadget $G^{*}$. The existence of a bipartite gadget with the same properties as $G^{*}$ would be a first step towards a proof that the problem 2-LIEC remains NP-complete when restricted to bipartite graphs. But we did not manage to find such a gadget so far. So we ask the following.

Question. Is 2-LIEC NP-complete when restricted to bipartite graphs? 


\section{References}

[1] L. Addario-Berry, R.E.L. Aldred, K. Dalal, and B.A. Reed. Vertex colouring edge partitions. J. Combin. Theory, Ser. B, 94(2):237 - 244, 2005 .

[2] Y. Alavi, G. Chartrand, F. R. K. Chung, P. Erdös, R. L. Graham, and O. R. Oellermann. How to define an irregular graph. J. Graph Theory, 11(2):235-249, 1987.

[3] O. Baudon, J. Bensmail, J. Przybyło, and M. Woźniak. On decomposing regular graphs into locally irregular subgraphs. Preprint MD 065, http://www.ii.uj.edu.pl/preMD/index.php, 2013.

[4] A. Dudek and D. Wajc. On the complexity of vertex-coloring edgeweightings. Discrete Math. Theor. Comput. Sci., 13(3):45-50, 2011.

[5] M. R. Garey and D. S. Johnson. Computers and Intractability: A Guide to the Theory of NP-Completeness. W. H. Freeman, 1979.

[6] F. Havet, N. Paramaguru, and R. Sampathkumar. Detection number of bipartite graphs and cubic graphs. Technical report, available at http://hal.archives-ouvertes.fr/hal-00744365/, 2012.

[7] M. Karoński, T. Łuczak, and A. Thomason. Edge weights and vertex colours. J. Combin. Theory, Ser. B, 91(1):151-157, 2004.

[8] B. Seamone. The 1-2-3 conjecture and related problems: a survey. Technical report, available at http://arxiv.org/abs/1211.5122, 2012.

[9] B. Seamone and B. Stevens. Sequence variations of the 1-2-3 conjecture and irregularity strength. Discrete Math. Theor. Comput. Sci., 15(1):15$28,2013$. 